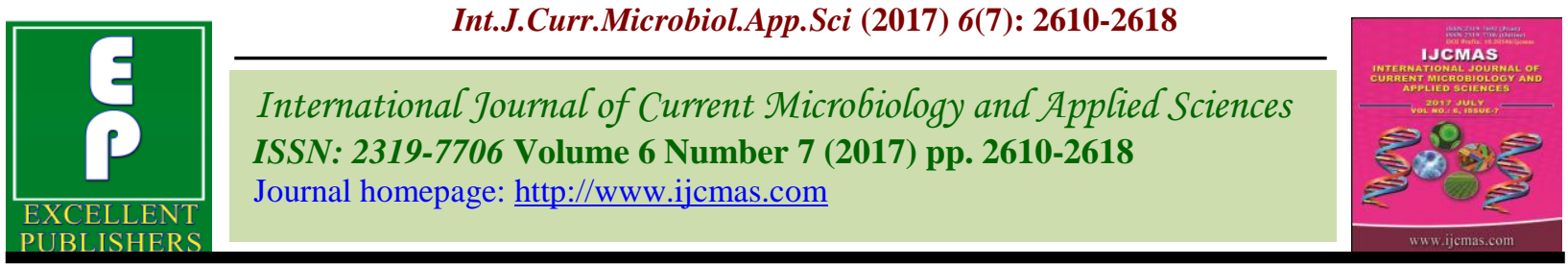

Original Research Article

https://doi.org/10.20546/ijcmas.2017.607.368

\title{
Variability in Grain Physico-Chemical Composition in Different Sorghum [Sorghum bicolor (L.) Moench] Genotypes
}

\author{
Chaitanya Itagi* and S. Hemalatha \\ Department of Food Science and Nutrition, College of Rural Home Science, University of \\ Agricultural Sciences, Dharwad - 580 005, Karnataka, India \\ *Corresponding author
}

\section{A B S T R A C T}

\begin{tabular}{|l|}
\hline Ke y w or d s \\
Chemical \\
composition, \\
Genotypes, Kharif, \\
Physical character, \\
Rabi.
\end{tabular}

The present investigation was carried out to study the physical characters such as hundred kernel weight, volume, density, colour, size and chemical composition such as moisture, protein, ash and minerals in different sorghum genotypes. The sorghum genotypes comprised of rabi local, advanced and released and kharif grains. There was significant variation in physical parameters such as hundred grain weight ( 2.19 to $4.33 \mathrm{~g}$ ), volume $(7.07$ to $8.40 \mathrm{ml})$, density $(0.29$ to 0.54 $\mathrm{g} / \mathrm{ml}$ ), colour and size. Similarly, chemical composition viz., moisture (4.39 to $9.29 \%$ ), protein (10.15 to $16.57 \%$ ), ash (1.53 to $9.29 \%)$ and mineral content like iron $(4.21$ to $18.37 \mathrm{mg} / 100 \mathrm{~g})$ and zinc $(0.44$ to $1.70 \mathrm{mg} / 100 \mathrm{~g})$ of different sorghum genotypes showed significant variation. Among all the sorghum genotypes, rabi genotypes had showed significant higher values of physicochemical composition compared to kharif genotypes except protein and zinc.

\section{Introduction}

Sorghum [Sorghum bicolor (L.) Moench] is an important staple food for more than 300 million people in Asia and Africa. Among the cereals, sorghum ranks fifth major cereal in terms of production and acreage in the world after wheat, maize, rice and barley. Sorghum has the potential for grain production even under low rainfall and they sustain adverse agro-climatic conditions. In India it is a staple food in the states of Maharashtra and parts of Karnataka, Madhya Pradesh, Tamil Nadu, Gujarat and Andhra Pradesh. Sorghum is cultivated in India during kharif (rainy) and rabi (post rainy) seasons. Sorghum is an excellent source of energy, contains $349 \mathrm{kcal}$, $10.4 \mathrm{~g}$ of protein, $1.9 \mathrm{~g}$ of fat and $72.6 \mathrm{~g}$ of carbohydrate and also has good amount of minerals particularly iron $(4.1 \mathrm{mg} / 100 \mathrm{~g})$ and zinc (1.6 mg/100g) (Gopalan et al., 2010). Modern genetic alterations are being used to modify cereals to increase their nutrient content. These approaches have the potential to have a major impact on the malnutrition of population groups who derive their dietary energy from a single cereal staple. Sorghum being a good source of nutrient a common food grain for most of the population, it can be well exploited for combating the deficiency of nutrients. Hence, better nutrient composition promotes nutritional empowerment of people dependent on sorghum as a staple food. Preliminary studies carried have shown that several genotypes in sorghum exhibit variation in nutrient content. 
Development of a variety with good nutrient content would help the breeders and consumers. Keeping view of this, the investigation was carried out on rabi and kharif sorghum genotypes with a specific a objective to screen the sorghum genotypes for grain physico-chemical composition.

\section{Materials and Methods}

The experiment was conducted during the year 2014-2015 at the department of Food Science and Nutrition, College of Rural Home Science, University of Agricultural Sciences, Dharwad. A total 24 sorghum grains of different genotypes were procured from All India Coordinated Research Project on Sorghum, University of Agricultural Sciences, Dharwad based on superior agronomic characteristics the genotypes were classified as local land races of rabi sorghum genotypes, released and advanced rabi and kharif genotypes.

Physical characteristics of grains of different sorghum genotypes such as 100 grain weight (g), volume (ml/100 grains), density (Mishra and Gupta, 1995), colour of grains was assessed in spectrophotometer model Konica Minolta, CM- 2600/2500d and was measured in chromatic components of ' $\mathrm{L}$ ' (black - 0 to white - 100), 'a' represents redness (+a values) to greenness (-a values) and ' $b$ ' represents yellowness ( $+b$ values) to blueness (-b values) and size of grains was recorded using digital caliper by measuring the dimensions at maximum points in 10 randomly selected grains $(\mathrm{mm})$.

Seeds of each sorghum genotype were powdered and analysed for chemical composition. Chemical composition such as moisture, crude protein, ash content by (Anon., 2010) and mineral contents such as iron and zinc were determined by atomic absorption spectrometry method.

\section{Results and Discussion}

The hundred-kernel weight of rabi sorghum genotypes viz., local land races, released and advanced and kharif sorghum genotypes showed significant $(\mathrm{p}<0.001)$ variation among all the genotypes and also within the genotype groups (Table 1). In local land races the hundred-kernel weight ranged between $2.46 \mathrm{~g}$ (Byahatti local) to $4.18 \mathrm{~g}$ (Lakmapur local). In case of rabi released and advanced sorghum genotypes hundred grain weight ranged between $2.49 \mathrm{~g}$ (Annigeri) and 4.33g (SVD0770). Whereas in kharif genotypes the kernel weight ranged between $2.19 \mathrm{~g}$ (SPV-2172) to $2.91 \mathrm{~g}$ (SPV-2250). Among the three different groups of sorghum genotypes, rabi released and advanced genotypes showed significant higher mean hundred-kernel weight (3.48 g). Hundred-kernel volume in local land races of rabi sorghum genotypes ranged from $7.43 \mathrm{ml}$ (Byahatti local) to $9.10 \mathrm{ml}$ (Lakmapur local). In rabi released and advanced sorghum genotypes volume ranged between $7.07 \mathrm{ml}$ (Muguti) to $8.40 \mathrm{ml}$ (SVD-0813) and in kharif sorghum genotypes ranged between 7.60 to $8.53 \mathrm{ml}$. On an average the hundred-kernel volume was highest in local rabi sorghum genotypes (Table 1). The hundred-kernel density of different rabi local sorghum genotypes ranged from $0.32 \mathrm{~g} / \mathrm{ml}$ (Giddamaldandi) to $0.51 \mathrm{~g} / \mathrm{ml}$ (Barsi jowar). Among rabi advanced and released genotypes, the least kernel density observed was found in Annigeri $(0.31 \mathrm{~g} / \mathrm{ml})$ and highest in SVD-0770 $(0.54 \mathrm{~g} / \mathrm{ml})$. Where as in kharif grown genotypes the kernel density ranged between $0.29 \mathrm{~g} / \mathrm{ml}$ (SPV-2172) to $0.34 \mathrm{~g} / \mathrm{ml}$ (SPV-2250). Rabi advanced and released sorghum genotypes showed higher bulk density compared to other genotypes. From the observations all the rabi genotypes showed higher kernel density $(0.54 \mathrm{~g} / \mathrm{ml})$ compared to kharif genotypes $(0.34 \mathrm{~g} / \mathrm{ml})$. The values of $\mathrm{L}, \mathrm{a}^{*}$ and $\mathrm{b}^{*}$ indicate, rabi released and advanced showed white chrome 
with yellow shades on it compared to other genotypes. When we compare colour of rabi and kharif grown sorghum genotype, rabi genotypes showed lighter chrome compared to kharif genotypes.

Length, width and breadth of the twenty four sorghum genotypes showed highly significant $(p<0.001)$ variation (Table 1). Length and width of the local genotypes of sorghum grains showed highest in Mudde mooti (4.94 and $4.23 \mathrm{~mm})$, Byahatti local had least breadth $(3.52 \mathrm{~mm})$. Released and advanced rabi genotypes SVD-0770 showed higher values of length $(4.96 \mathrm{~mm})$, width $(4.27 \mathrm{~mm})$ and breadth $(3.03 \mathrm{~mm})$. In kharif genotypes SPV-2250 showed higher length $(4.55 \mathrm{~mm})$, width $(3.52 \mathrm{~mm})$ and breadth $(2.43 \mathrm{~mm})$. Compared to kharif, rabi genotypes showed significantly higher values of length, width and breadth. Samdhur et al., (2013) observed wide variations in hundred-kernel weight, volume and density among rabi and kharif grown sorghum genotypes. These variations might be due to the influence of genetic and environmental factors.

Moisture content in grains of sorghum genotypes are presented in table 2. Moisture content in local rabi sorghum genotypes varied significantly $(\mathrm{p}<0.001)$ and ranged between 4.39 (Giddamaldandi) and 9.29 per cent (Madabavi local). In rabi advanced and released genotypes moisture content ranged between 4.69 (SPV-2217) and 8.10 per cent (SVD-0765). In kharif grown genotypes the range was between 5.61 (SPV-2172) and 5.89 per cent (SPV-2250). Comparatively, rabi local land races showed significant higher mean moisture $(6.94 \%)$ content than those of rabi released and advanced and kharif grown genotypes. The moisture content of sorghum genotypes almost close to the values (between 8.3 to $8.43 \%$ ) reported by Gassem and Osman (2003).

Crude protein (Table 2) content of local land races of rabi sorghum genotypes ranged between 10.15 (Byahatti local) and 16.57 per cent (Ramke local) with a significant $(\mathrm{p}<0.001)$ variation. In rabi released and advanced genotypes the protein content ranged between 10.95 (Muguti) and 14.12 per cent (SVD-0765). In kharif genotypes protein content was least in SPV-2250 (14.57 \%) and highest in SPV-2172 (15.52 \%). The protein mean content was highest in sorghum grown in kharif $(16.04 \%)$ than in rabi genotypes (Fig 1). Geeta et al., (2005) reported that the physico-chemical characterization of sorghum accessions showed a wide variation in protein (7.99 to $17.8 \%$ ) and reasoned that wide variation was due to influence of environmental condition.

Fig.1 Mean protein (\%), ash (\%), iron (mg/100g) and zinc (mg/100g) content in sorghum grains of rabi local land races, rabi released and advanced and kharif genotypes

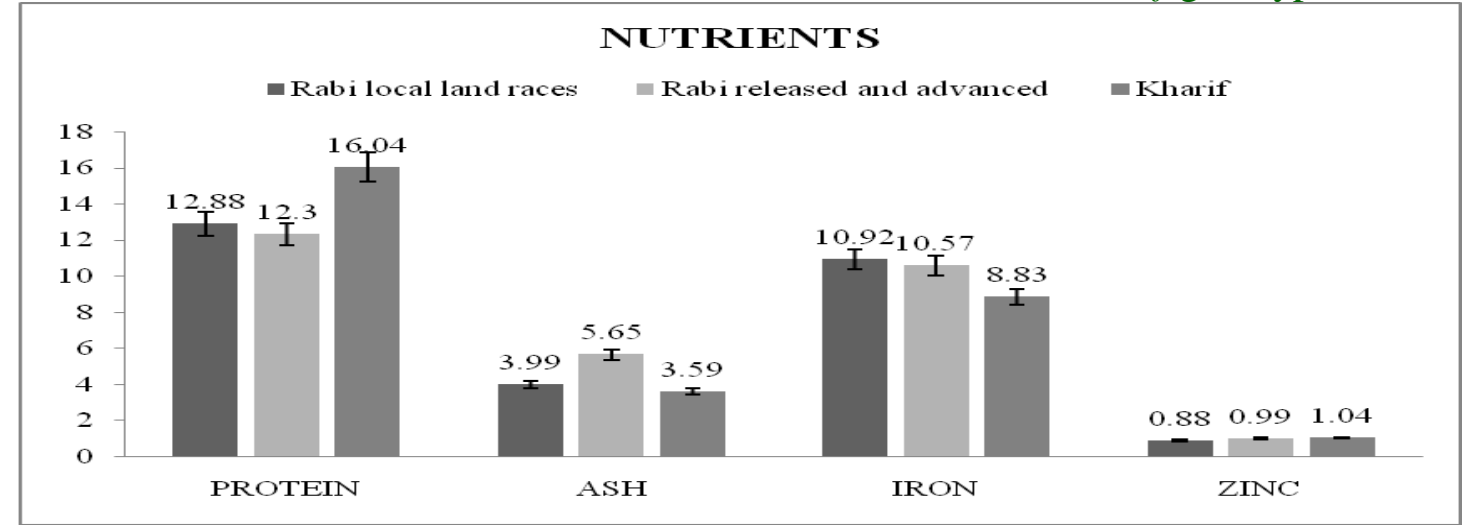


Table.1 Physical characteristics of grains of sorghum genotypes

\begin{tabular}{|c|c|c|c|c|c|c|c|c|c|}
\hline Genotypes & $\begin{array}{l}100 \text { kernel } \\
\text { weight (g) }\end{array}$ & $\begin{array}{l}\text { Volume } \\
\text { (ml) }\end{array}$ & $\begin{array}{c}\text { Density } \\
\text { (g/ml) }\end{array}$ & $\mathbf{L}^{*}$ & $\mathbf{a}^{*}$ & $\mathbf{b}^{*}$ & $\begin{array}{l}\text { Grain } \\
\text { length } \\
(\mathbf{m m})\end{array}$ & $\begin{array}{l}\text { Grain width } \\
\quad(\mathbf{m m})\end{array}$ & $\begin{array}{l}\text { Grain } \\
\text { breadth } \\
(\mathrm{mm})\end{array}$ \\
\hline \multicolumn{10}{|c|}{ Local land races of rabi sorghum genotypes } \\
\hline Lakmapur local & $4.18 \pm 0.14^{\mathrm{a}}$ & $9.10 \pm 0.10^{\mathrm{a}}$ & $0.46 \pm 0.02^{b}$ & $86.29 \pm 0.10^{\mathrm{de}}$ & $1.34 \pm 0.04^{\mathrm{g}}$ & $12.23 \pm 0.18^{\mathrm{bc}}$ & $4.42 \pm 0.29^{\mathrm{de}}$ & $4.12 \pm 0.24^{\mathrm{abc}}$ & $2.63 \pm 0.24^{b c}$ \\
\hline Barsi zoot & $3.29 \pm 0.04^{c}$ & $8.17 \pm 0.15^{c}$ & $0.40 \pm 0.01^{\mathrm{c}}$ & $83.88 \pm 0.06^{\mathrm{bc}}$ & $2.30 \pm 0.01^{\mathrm{d}}$ & $11.48 \pm 0.04^{\mathrm{ef}}$ & $4.65 \pm 0.31^{\mathrm{cd}}$ & $3.82 \pm 0.23^{\mathrm{de}}$ & $2.62 \pm 0.23^{\mathrm{cd}}$ \\
\hline Ramke local & $3.68 \pm 0.09^{\mathrm{b}}$ & $8.10 \pm 0.17^{\mathrm{c}}$ & $0.45 \pm 0.02^{\mathrm{b}}$ & $85.85 \pm 0.08^{\mathrm{abc}}$ & $1.87 \pm 0.02^{\mathrm{d}}$ & $12.4 \pm 0.014^{\mathrm{ab}}$ & $4.82 \pm 0.15^{\mathrm{abc}}$ & $3.92 \pm 0.26^{\text {bcde }}$ & $2.91 \pm 0.26^{\mathrm{a}}$ \\
\hline Kodamurki & $3.38 \pm 0.01^{\mathrm{c}}$ & $8.20 \pm 0.10^{\mathrm{c}}$ & $0.41 \pm 0.01^{\mathrm{c}}$ & $86.49 \pm 0.05^{\mathrm{de}}$ & $1.67 \pm 0.04^{\mathrm{e}}$ & $12.40 \pm 0.15^{\mathrm{ab}}$ & $4.55 \pm 0.27^{\mathrm{de}}$ & $3.90 \pm 0.40^{\text {cde }}$ & $2.85 \pm 0.40^{\mathrm{ab}}$ \\
\hline Madabavi local & $3.29 \pm 0.05^{\mathrm{c}}$ & $7.53 \pm 0.06^{\mathrm{d}}$ & $0.44 \pm 0.00^{\mathrm{b}}$ & $84.40 \pm 0.06^{\mathrm{de}}$ & $1.96 \pm 0.02^{\mathrm{h}}$ & $11.19 \pm 0.17^{\mathrm{f}}$ & $4.46 \pm 0.24^{\mathrm{de}}$ & $3.89 \pm 0.07^{\text {cde }}$ & $2.68 \pm 0.07^{\mathrm{bcd}}$ \\
\hline Chitapur local & $3.04 \pm 0.15^{\mathrm{d}}$ & $7.47 \pm 0.14^{\mathrm{d}}$ & $0.41 \pm 0.03^{c}$ & $85.05 \pm 0.04^{\mathrm{cd}}$ & $1.24 \pm 0.02^{\mathrm{e}}$ & $10.65 \pm 0.13^{\mathrm{g}}$ & $4.60 \pm 0.18^{\mathrm{cd}}$ & $3.84 \pm 0.10^{\mathrm{de}}$ & $2.61 \pm 0.09^{\text {cde }}$ \\
\hline Byahatti local & $2.46 \pm 0.23^{\mathrm{f}}$ & $7.43 \pm 0.12^{\mathrm{d}}$ & $0.33 \pm 0.03^{\mathrm{e}}$ & $81.62 \pm 0.23^{f}$ & $1.66 \pm 0.03^{\mathrm{f}}$ & $12.49 \pm 0.31^{\mathrm{ab}}$ & $4.10 \pm 0.21^{\mathrm{f}}$ & $3.70 \pm 0.20^{\mathrm{e}}$ & $3.52 \pm 0.19^{\mathrm{def}}$ \\
\hline Barsi jowar & $4.16 \pm 0.09^{\mathrm{a}}$ & $8.23 \pm 0.12^{\mathrm{c}}$ & $0.51 \pm 0.01^{\mathrm{a}}$ & $86.56 \pm 0.31^{\mathrm{ab}}$ & $1.49 \pm 0.11^{\mathrm{d}}$ & $11.78 \pm 0.30^{\mathrm{de}}$ & $4.85 \pm 0.19^{\mathrm{ab}}$ & $4.14 \pm 0.26^{\mathrm{ab}}$ & $3.03 \pm 0.26^{\mathrm{ef}}$ \\
\hline Tansalwadi & $2.86 \pm 0.07^{\mathrm{de}}$ & $8.13 \pm 0.15^{c}$ & $0.35 \pm 0.01^{\mathrm{d}}$ & $86.45 \pm 0.16^{\mathrm{de}}$ & $1.82 \pm 0.07^{\mathrm{d}}$ & $12.68 \pm 0.26^{\mathrm{a}}$ & $4.47 \pm 0.34^{\mathrm{de}}$ & $4.06 \pm 0.23^{\mathrm{ab}}$ & $2.51 \pm 0.23^{\text {cdef }}$ \\
\hline Mudde mooti & $3.06 \pm 0.13^{\mathrm{d}}$ & $7.50 \pm 0.15^{\mathrm{d}}$ & $0.41 \pm 0.02^{c}$ & $85.76 \pm 0.04^{\mathrm{a}}$ & $2.05 \pm 0.04^{\mathrm{b}}$ & $12.24 \pm 0.13^{b c}$ & $4.94 \pm 0.22^{\mathrm{a}}$ & $4.23 \pm 0.20 \mathrm{a}$ & $3.01 \pm 0.20^{\mathrm{f}}$ \\
\hline Giddamaladandi & $2.74 \pm 0.07^{\mathrm{e}}$ & $8.53 \pm 0.15^{b}$ & $0.32 \pm 0.01^{\mathrm{e}}$ & $85.05 \pm 0.03^{\mathrm{e}}$ & $1.89 \pm 0.04^{\mathrm{cd}}$ & $11.93 \pm 0.04^{\mathrm{cd}}$ & $4.33 \pm 0.28^{\mathrm{e}}$ & $3.82 \pm 0.26^{\mathrm{de}}$ & $2.56 \pm 0.26^{\text {cdef }}$ \\
\hline Mean \pm SD & $3.28 \pm 0.53$ & $8.03 \pm 0.51^{\mathrm{d}}$ & $0.41 \pm 0.05$ & $85.21 \pm 1.44$ & $1.75 \pm 0.31$ & $11.95 \pm 0.62$ & $4.56 \pm 0.33$ & $3.98 \pm 0.27$ & $2.69 \pm 0.23$ \\
\hline F Value & $69.5^{* * *}$ & $52.28 * * *$ & $31.72 * * *$ & $361.83 * * *$ & $130.61 * * *$ & $34.48 * * *$ & $9.79 * * *$ & $4.81 * * *$ & $7.66^{* * *}$ \\
\hline SEM \pm & 0.065 & 0.07 & 0.02 & 0.07 & 0.03 & 0. & 0.14 & 0.13 & 0.10 \\
\hline $\mathrm{CD}$ at $0.1 \%$ & 0.19 & 0.21 & 0.06 & 0.23 & 0.07 & 0.31 & 0.40 & 0.38 & 0.29 \\
\hline \multicolumn{10}{|c|}{ Released and advanced rabi sorghum genotypes } \\
\hline Muguti & $3.73 \pm 0.08^{c}$ & $7.07 \pm 0.12^{\mathrm{d}}$ & $0.53 \pm 0.01^{\mathrm{a}}$ & $85.29 \pm 0.26^{\mathrm{e}}$ & $1.87 \pm 0.04^{\mathrm{b}}$ & $11.76 \pm 0.04^{\mathrm{d}}$ & $4.78 \pm 0.26^{\mathrm{abc}}$ & $4.13 \pm 0.26^{\mathrm{ab}}$ & $2.82 \pm 0.26^{\mathrm{bc}}$ \\
\hline SVD-0770 & $4.33 \pm 0.05^{\mathrm{a}}$ & $8.17 \pm 0.15^{\mathrm{ab}}$ & $0.54 \pm 0.01^{\mathrm{a}}$ & $84.24 \pm 0.08^{\mathrm{g}}$ & $1.45 \pm 0.03^{\mathrm{e}}$ & $11.04 \pm 0.02^{\mathrm{f}}$ & $4.96 \pm 0.23^{\mathrm{a}}$ & $4.27 \pm 0.16^{\mathrm{a}}$ & $3.03 \pm 0.16^{\mathrm{a}}$ \\
\hline SVD - 0813 & $3.55 \pm 0.10^{\mathrm{d}}$ & $8.40 \pm 0.27^{\mathrm{a}}$ & $0.43 \pm 0.01^{\mathrm{c}}$ & $84.77 \pm 0.13^{f}$ & $1.58 \pm 0.03^{\mathrm{d}}$ & $11.19 \pm 0.02^{\mathrm{f}}$ & $4.67 \pm 0.29^{\mathrm{bcd}}$ & $4.03 \pm 0.20^{\mathrm{b}}$ & $2.72 \pm 0.16^{\mathrm{cd}}$ \\
\hline M35 - 1 & $3.33 \pm 0.07^{\mathrm{f}}$ & $8.07 \pm 0.21^{b}$ & $0.41 \pm 0.01^{\mathrm{d}}$ & $86.83 \pm 0.05^{\mathrm{c}}$ & $1.81 \pm 0.04^{\mathrm{b}}$ & $11.70 \pm 0.24^{\mathrm{de}}$ & $4.53 \pm 0.18^{\mathrm{d}}$ & $3.91 \pm 0.23^{b}$ & $2.7 \pm 0.23^{\mathrm{cd}}$ \\
\hline
\end{tabular}




\begin{tabular}{|c|c|c|c|c|c|c|c|c|c|}
\hline SVD -0765 & $3.44 \pm 0.02^{\mathrm{ef}}$ & $8.20 \pm 0.20^{\mathrm{ab}}$ & $0.42 \pm 0.01^{\mathrm{cd}}$ & $84.47 \pm 0.10^{\mathrm{g}}$ & $1.60 \pm 0.12^{\mathrm{d}}$ & $11.56 \pm 0.07^{\mathrm{e}}$ & $4.53 \pm 0.19^{\mathrm{d}}$ & $4.04 \pm 0.22^{\mathrm{c}}$ & $2.72 \pm 0.22^{c}$ \\
\hline SVD - 0808 & $3.89 \pm 0.03^{b}$ & $7.53 \pm 0.06^{\mathrm{c}}$ & $0.52 \pm 0.00^{\mathrm{a}}$ & $87.75 \pm 0.35^{a}$ & $1.24 \pm 0.03^{\mathrm{f}}$ & $11.85 \pm 0.14^{\mathrm{d}}$ & $4.53 \pm 0.16^{\mathrm{d}}$ & $4.27 \pm 0.10^{\mathrm{a}}$ & $2.67 \pm 0.10^{\mathrm{cd}}$ \\
\hline DSV -4 & $2.93 \pm 0.06^{\mathrm{g}}$ & $8.13 \pm 0.12^{\mathrm{ab}}$ & $0.36 \pm 0.01^{\mathrm{e}}$ & $83.79 \pm 0.03^{h}$ & $2.56 \pm 0.02^{\mathrm{a}}$ & $14.10 \pm 0.07^{\mathrm{a}}$ & $4.25 \pm 0.32^{\mathrm{e}}$ & $3.97 \pm 0.20^{\mathrm{b}}$ & $2.32 \pm 0.20^{f}$ \\
\hline IS - 4698 & $3.79 \pm 0.07^{\mathrm{bc}}$ & $7.97 \pm 0.06^{\mathrm{b}}$ & $0.48 \pm 0.01^{\mathrm{b}}$ & $85.22 \pm 0.09^{\mathrm{e}}$ & $1.64 \pm 0.27^{\mathrm{cd}}$ & $13.00 \pm 0.05^{\mathrm{b}}$ & $4.89 \pm 0.21^{\mathrm{ab}}$ & $4.27 \pm 0.10^{\mathrm{a}}$ & $2.95 \pm 0.10 \mathrm{ab}$ \\
\hline $\mathrm{DSV}-5$ & $3.34 \pm 0.05^{\mathrm{f}}$ & $8.27 \pm 0.21^{\mathrm{ab}}$ & $0.40 \pm 0.02^{\mathrm{d}}$ & $87.20 \pm 0.16^{\mathrm{c}}$ & $1.72 \pm 0.06^{\mathrm{c}}$ & $12.42 \pm 0.06^{\mathrm{c}}$ & $4.57 \pm 0.28^{\mathrm{cd}}$ & $4.10 \pm 0.30^{\mathrm{ab}}$ & $2.49 \pm 0.27^{\mathrm{e}}$ \\
\hline SPV - 2217 & $3.50 \pm 0.08^{\mathrm{de}}$ & $7.53 \pm 0.15^{c}$ & $0.47 \pm 0.02^{b}$ & $86.45 \pm 0.07^{\mathrm{d}}$ & $1.65 \pm 0.03^{\mathrm{cd}}$ & $11.81 \pm 0.08^{\mathrm{d}}$ & $4.54 \pm 0.18^{\mathrm{d}}$ & $4.28 \pm 0.20^{\mathrm{a}}$ & $2.68 \pm 0.20^{\mathrm{de}}$ \\
\hline Annigeri & $2.49 \pm 0.03^{\mathrm{h}}$ & $7.60 \pm 0.20^{c}$ & $0.31 \pm 0.01^{\mathrm{f}}$ & $86.25 \pm 0.08^{\mathrm{d}}$ & $1.61 \pm 0.02^{\mathrm{d}}$ & $11.17 \pm 0.07^{\mathrm{f}}$ & $3.94 \pm 0.30^{\mathrm{f}}$ & $3.58 \pm 0.40 \mathrm{c}$ & $2.42 \pm 0.40^{\mathrm{f}}$ \\
\hline Mean \pm SD & $3.48 \pm 0.47$ & $7.90 \pm 0.42$ & $0.44 \pm 0.06$ & $85.66 \pm 1.27$ & $1.70 \pm 0.32$ & $11.96 \pm 0.88$ & $4.56 \pm 0.37$ & $4.08 \pm 0.30$ & $2.66 \pm 0.27$ \\
\hline F Value & $189.28 * * *$ & $17.56 * * *$ & $119.76 * * *$ & $212.8 * * *$ & $141.15^{* * *}$ & $263.7 * * *$ & $14.78 * * *$ & $9.96 * * *$ & $17.57 * * *$ \\
\hline SEM \pm & 0.04 & 0.09 & 0.02 & 0.08 & 0.03 & 0.05 & 0.14 & 0.12 & 0.10 \\
\hline $\mathrm{CD}$ at $0.1 \%$ & 0.11 & 0.28 & 0.05 & 0.26 & 0.07 & 0.16 & 0.40 & 0.36 & 0.30 \\
\hline \multicolumn{10}{|c|}{ Kharif sorghum genotypes } \\
\hline SPV-2172 & $2.19 \pm 0.08$ & $7.60 \pm 0.10$ & $0.29 \pm 0.01$ & $85.14 \pm 0.03$ & $1.56 \pm 0.03$ & $12.40 \pm 0.02$ & $3.83 \pm 0.22$ & $3.28 \pm 0.17$ & $2.44 \pm 0.17$ \\
\hline$S P V-2250$ & $2.91 \pm 0.05$ & $8.53 \pm 0.15$ & $0.34 \pm 0.01$ & $85.23 \pm 0.06$ & $1.50 \pm 0.02$ & $12.25 \pm 0.12$ & $4.55 \pm 0.30$ & $3.52 \pm 0.24$ & $2.43 \pm 0.24$ \\
\hline Mean \pm SD & $2.55 \pm 0.39$ & $8.06 \pm 0.52$ & $0.313 \pm 0.03$ & $85.18 \pm 0.06$ & $1.53 \pm 0.03$ & $12.32 \pm 0.11$ & $4.18 \pm 0.44$ & $3.39 \pm 0.23$ & $2.62 \pm 0.29$ \\
\hline F Value & $180.12 * * *$ & $78.4 * * *$ & $74.79 * * *$ & $5.63 \mathrm{NS}$ & $6.76 \mathrm{NS}$ & $4.23 \mathrm{NS}$ & $36.25 * * *$ & $6.24 *$ & $14.65 * * *$ \\
\hline SEM \pm & 0.04 & 0.08 & 0.07 & 0.03 & 0.02 & 0.05 & 0.15 & 0.11 & 0.13 \\
\hline $\mathrm{CD}$ at $0.1 \%$ & 0.12 & 0.24 & 0.23 & 0.10 & 0.07 & 0.18 & 0.45 & 0.34 & 0.39 \\
\hline \multicolumn{10}{|c|}{ Over all } \\
\hline Mean \pm SD & $3.32 \pm 0.55$ & $7 . .97 \pm 047$ & $0.42 \pm 0.07$ & $85.41 \pm 1.31$ & $1.71 \pm 0.30$ & $11.98 \pm 0.73$ & $2.53+0.36$ & $3.96+0.32$ & $2.59+032$ \\
\hline F Value & $116.36^{* * *}$ & $29.69 * * *$ & $73.12 * * *$ & $268.85^{* * *}$ & $131.63^{* * *}$ & $79.32 * * *$ & $13.63 * * *$ & $12.77 * * *$ & $9.77 * * *$ \\
\hline SEM \pm & 0.05 & 0.08 & 0.02 & 0.08 & 0.03 & 0.08 & 0.04 & 0.05 & 0.05 \\
\hline $\mathrm{CD}$ at $0.1 \%$ & 0.14 & 0.24 & 0.06 & 0.23 & 0.07 & 0.24 & 0.13 & 0.13 & 0.13 \\
\hline
\end{tabular}

CD- Critical Difference

$* * *$ The values are significantly different at $\mathrm{p}<0.001$, NS-non significant, All values are calculated in triplicate and are expressed in terms of means \pm SD 
Table.2 Moisture, protein and ash content in grains of different sorghum genotypes

\begin{tabular}{|c|c|c|c|}
\hline $\begin{array}{ll}\text { Genotypes } & \text { Nutrients } \\
\end{array}$ & $\begin{array}{c}\text { Moisture } \\
(\%)\end{array}$ & $\begin{array}{c}\text { Protein } \\
(\%)\end{array}$ & $\begin{array}{l}\text { Ash } \\
(\%)\end{array}$ \\
\hline \multicolumn{4}{|c|}{ Local land races of rabi sorghum genotypes } \\
\hline Lakmapur local & $6.58 \pm 0.16^{\mathrm{e}}$ & $14.23 \pm 0.53^{\mathrm{ab}}$ & $1.53 \pm 0.13^{\mathrm{g}}$ \\
\hline Barsi zoot & $4.94 \pm 0.11^{\mathrm{g}}$ & $11.84 \pm 0.79^{\mathrm{cd}}$ & $1.57 \pm 0.03^{\mathrm{tg}}$ \\
\hline Ramke local & $7.77 \pm 0.28^{\mathrm{cd}}$ & $16.57 \pm 1.10^{\mathrm{a}}$ & $7.78 \pm 0.28^{\mathrm{d}}$ \\
\hline Kodamurki & $7.38 \pm 0.37^{\mathrm{d}}$ & $11.32 \pm 1.76^{\mathrm{cd}}$ & $1.81 \pm 0.02^{\mathrm{e}}$ \\
\hline Madabavi local & $9.29 \pm 0.11^{\mathrm{a}}$ & $13.88 \pm 1.46^{\mathrm{ab}}$ & $9.29 \pm 0.11^{\mathrm{a}}$ \\
\hline Chitapur local & $6.85 \pm 0.03^{\mathrm{e}}$ & $11.67 \pm 0.72^{\mathrm{cd}}$ & $6.85 \pm 0.03^{\mathrm{d}}$ \\
\hline Byahatti local & $7.96 \pm 0.62^{\mathrm{c}}$ & $10.15 \pm 1.81^{\mathrm{d}}$ & $1.61 \pm 0.15^{\mathrm{efg}}$ \\
\hline Barsi jowar & $5.96 \pm 0.36^{\mathrm{f}}$ & $14.35 \pm 0.35^{\mathrm{ab}}$ & $1.64 \pm 0.04^{\mathrm{ef}}$ \\
\hline Tansalwadi & $6.71 \pm 0.11^{\mathrm{e}}$ & $14.58 \pm 0.53^{\mathrm{a}}$ & $1.79 \pm 0.08^{\mathrm{ef}}$ \\
\hline Mudde mooti & $8.55 \pm 0.14^{b}$ & $11.73 \pm 1.14^{\mathrm{cd}}$ & $8.55 \pm 0.14^{b}$ \\
\hline Giddamaladandi & $4.39 \pm 0.26^{\mathrm{g}}$ & $12.48 \pm 0.53^{\mathrm{bc}}$ & $1.56 \pm 0.07^{\mathrm{g}}$ \\
\hline Mean \pm SD & $6.94 \pm 1.44$ & $12.88 \pm 1.87$ & $3.99 \pm 3.21$ \\
\hline F Value & $81.7 * * *$ & $8.5 * * *$ & $2.21 * * *$ \\
\hline SEM \pm & 0.30 & 0.60 & 0.41 \\
\hline $\mathrm{CD}$ at $0.1 \%$ & 0.91 & 1.81 & 1.23 \\
\hline \multicolumn{4}{|c|}{ Released and advanced rabi sorghum genotypes } \\
\hline Muguti & $6.31 \pm 0.46^{b}$ & $10.95 \pm 0.51^{\mathrm{c}}$ & $6.32 \pm 0.46^{b}$ \\
\hline SVD-0770 & $7.83 \pm 0.39^{\mathrm{a}}$ & $11.78 \pm 0.53^{\mathrm{bc}}$ & $7.83 \pm 0.39^{\mathrm{a}}$ \\
\hline SVD - 0813 & $6.21 \pm 0.61^{b}$ & $11.90 \pm 0.35^{\mathrm{bc}}$ & $1.64 \pm 0.02^{\mathrm{d}}$ \\
\hline M 35-1 & $6.76 \pm 0.23^{b}$ & $12.43 \pm 1.06^{\mathrm{b}}$ & $6.76 \pm 0.23^{b}$ \\
\hline SVD - 0765 & $8.10 \pm 0.18^{\mathrm{a}}$ & $14.12 \pm 0.53^{\mathrm{a}}$ & $8.10 \pm 0.18^{\mathrm{a}}$ \\
\hline SVD - 0808 & $5.24 \pm 0.99^{c}$ & $11.65 \pm 0.54^{\mathrm{bc}}$ & $5.24 \pm 0.99^{\mathrm{c}}$ \\
\hline DSV -4 & $6.83 \pm 0.09^{b}$ & $12.13 \pm 0.88^{\mathrm{bc}}$ & $6.83 \pm 0.09^{b}$ \\
\hline IS - 4698 & $6.40 \pm 0.52^{b}$ & $14.00 \pm 0.35^{\mathrm{a}}$ & $6.40 \pm 0.52^{\mathrm{b}}$ \\
\hline $\mathrm{DSV}-5$ & $5.28 \pm 0.37^{\mathrm{C}}$ & $12.25 \pm 0.35^{\mathrm{b}}$ & $1.60 \pm 0.04^{\mathrm{c}}$ \\
\hline SPV - 2217 & $4.69 \pm 0.41^{\mathrm{c}}$ & $12.37 \pm 0.72^{\mathrm{b}}$ & $4.69 \pm 0.41^{\mathrm{b}}$ \\
\hline Annigeri & $6.76 \pm 0.18^{b}$ & $12.72 \pm 0.72^{b}$ & $6.76 \pm 0.18^{b}$ \\
\hline Mean \pm SD & $6.40 \pm 1.08$ & $12.30 \pm 1.05$ & $5.65 \pm 2.17$ \\
\hline F Value & $14.84 * * *$ & $6.66 * * *$ & $84.56 * * *$ \\
\hline SEM \pm & 0.25 & 0.31 & 0.35 \\
\hline $\mathrm{CD}$ at $0.1 \%$ & 0.70 & 0.90 & 0.90 \\
\hline \multicolumn{4}{|c|}{ Kharif sorghum genotypes } \\
\hline SPV-2172 & $5.61 \pm 0.08$ & $15.52 \pm 0.40$ & $5.61 \pm 0.08$ \\
\hline SPV -2250 & $5.89 \pm 0.24$ & $14.57 \pm 0.88$ & $1.60 \pm 0.07$ \\
\hline Mean \pm SD & $5.75 \pm 0.22$ & $16.04 \pm 0.84$ & $3.59 \pm 2.21$ \\
\hline F Value & $3.53^{\mathrm{NS}}$ & $3.52^{\mathrm{NS}}$ & $42.8^{* * * *}$ \\
\hline SEM \pm & 0.10 & 0.39 & 0.04 \\
\hline $\mathrm{CD}$ at $0.1 \%$ & 0.30 & 1.25 & 0.13 \\
\hline \multicolumn{4}{|c|}{ Over all } \\
\hline Mean \pm SD & $6.59 \pm 1.26$ & $12.92 \pm 1.75$ & $4.72 \pm 2.80$ \\
\hline F Value & $33.26 * * *$ & $10.30 * * *$ & $276.51 * * *$ \\
\hline SEM \pm & 0.21 & 0.263 & 0.17 \\
\hline $\mathrm{CD}$ at $0.1 \%$ & 0.61 & 0.747 & 0.48 \\
\hline
\end{tabular}

CD- Critical Difference, $(\mathrm{a}-\mathrm{g})$ Different letters as superscript denote significant differences $(\mathrm{p}<0.001)$ between moisture $(\%)$, protein $(\%)$ and ash $(\%)$ content in grains of different sorghum genotypes, NS- non-significant, All values are calculated in triplicate and are expressed in terms of means \pm SD 
Table.3 Iron and zinc content in grains of sorghum genotypes

\begin{tabular}{|c|c|c|}
\hline $\begin{array}{ll}\text { Genotypes } & \text { Minerals } \\
\end{array}$ & $\begin{array}{c}\text { Iron } \\
(\mathrm{mg} / 100 \mathrm{~g})\end{array}$ & $\begin{array}{c}\text { Zinc } \\
(\mathrm{mg} / 100 \mathrm{~g})\end{array}$ \\
\hline \multicolumn{3}{|c|}{ Local land races of rabi sorghum genotypes } \\
\hline Lakmapur local & $4.99 \pm 0.34^{\mathrm{h}}$ & $1.10 \pm 0.60^{\mathrm{b}}$ \\
\hline Barsi zoot & $7.95 \pm 0.72^{\mathrm{g}}$ & $1.06 \pm 0.03^{\mathrm{b}}$ \\
\hline Ramke local & $9.18 \pm 0.40^{\mathrm{f}}$ & $0.45 \pm 0.05^{\mathrm{d}}$ \\
\hline Kodamurki & $9.29 \pm 0.55^{\mathrm{f}}$ & $1.70 \pm 0.04^{\mathrm{a}}$ \\
\hline Madabavi local & $9.30 \pm 0.63^{\mathrm{f}}$ & $0.44 \pm 0.02^{\mathrm{d}}$ \\
\hline Chitapur local & $10.06 \pm 0.70^{\mathrm{ef}}$ & $0.69 \pm 0.05^{\mathrm{d}}$ \\
\hline Byahatti local & $10.99 \pm 0.24^{\mathrm{de}}$ & $0.97 \pm 0.03^{\mathrm{d}}$ \\
\hline Barsi jowar & $11.58 \pm 0.66^{\mathrm{cd}}$ & $1.28 \pm 0.02^{\mathrm{b}}$ \\
\hline Tansalwadi & $12.45 \pm 0.68^{\mathrm{c}}$ & $0.45 \pm 0.03^{\mathrm{d}}$ \\
\hline Mudde mooti & $16.08 \pm 0.10^{\mathrm{b}}$ & $0.50 \pm 0.03^{\mathrm{d}}$ \\
\hline Giddamaladandi & $18.37 \pm 0.80^{\mathrm{a}}$ & $1.09 \pm 0.02^{\mathrm{b}}$ \\
\hline Mean \pm SD & $10.92 \pm 3.62$ & $0.88 \pm 0.42$ \\
\hline F Value & $127.66 * * *$ & $15.24 * * *$ \\
\hline SEM \pm & 0.33 & 0.11 \\
\hline $\mathrm{CD}$ at $0.1 \%$ & 0.96 & 0.31 \\
\hline \multicolumn{3}{|c|}{ Released and advanced rabi sorghum genotypes } \\
\hline Muguti & $4.21 \pm 0.23^{\mathrm{e}}$ & $1.38 \pm 0.14^{\mathrm{c}}$ \\
\hline SVD-0770 & $4.53 \pm 0.80^{\mathrm{e}}$ & $0.49 \pm 0.02^{\mathrm{h}}$ \\
\hline SVD -0813 & $6.99 \pm 0.56^{\mathrm{d}}$ & $0.70 \pm 0.02^{\mathrm{f}}$ \\
\hline M 35-1 & $9.62 \pm 0.92^{\mathrm{c}}$ & $1.63 \pm 0.03^{\mathrm{a}}$ \\
\hline SVD - 0765 & $10.79 \pm 1.80^{\mathrm{bc}}$ & $0.59 \pm 0.01^{\mathrm{g}}$ \\
\hline SVD -0808 & $11.33 \pm 1.66^{\mathrm{bc}}$ & $0.78 \pm 0.01^{\mathrm{e}}$ \\
\hline DSV -4 & $12.33 \pm 1.46^{\mathrm{b}}$ & $0.85 \pm 0.02^{\mathrm{e}}$ \\
\hline IS - 4698 & $12.70 \pm 0.79^{b}$ & $1.32 \pm 0.04^{\mathrm{d}}$ \\
\hline DSV -5 & $12.70 \pm 1.12^{\mathrm{b}}$ & $0.62 \pm 0.02^{\mathrm{g}}$ \\
\hline SPV -2217 & $14.71 \pm 0.71^{\mathrm{a}}$ & $1.49 \pm 0.03^{\mathrm{b}}$ \\
\hline Annigeri & $16.43 \pm 1.03^{\mathrm{a}}$ & $1.13 \pm 0.03^{\mathrm{e}}$ \\
\hline Mean \pm SD & $10.57 \pm 3.92$ & $0.99 \pm 0.39$ \\
\hline F Value & $38.15 * * *$ & $228.27 * * *$ \\
\hline SEM \pm & 0.63 & 0.03 \\
\hline $\mathrm{CD}$ at $0.1 \%$ & 1.87 & 0.08 \\
\hline \multicolumn{3}{|c|}{ Kharif sorghum genotypes } \\
\hline SPV-2172 & $4.82 \pm 0.70$ & $0.84 \pm 0.01$ \\
\hline SPV - 2250 & $12.85 \pm 0.25$ & $1.25 \pm 0.04$ \\
\hline Mean \pm SD & $8.83 \pm 4.42$ & $1.04 \pm 0.22$ \\
\hline F Value & $350.20 * * *$ & $335.90 * * *$ \\
\hline SEM \pm & 0.30 & 0.02 \\
\hline $\mathrm{CD}$ at $0.1 \%$ & 1.09 & 0.07 \\
\hline \multicolumn{3}{|c|}{ Over all } \\
\hline Mean \pm SD & $10.59 \pm 3.80$ & $0.95 \pm 0.39$ \\
\hline F Value & $59.41 * * *$ & $27.87 * * *$ \\
\hline SEM \pm & 0.49 & 0.07 \\
\hline $\mathrm{CD}$ at $0.1 \%$ & 1.40 & 0.21 \\
\hline
\end{tabular}

CD- Critical Difference, (a-e) Different letters as superscript denote significant differences $(\mathrm{p}<0.001)$ between iron $(\mathrm{mg} / 100 \mathrm{~g})$ and zinc $(\mathrm{mg} / 100 \mathrm{~g})$ content in grains of different sorghum genotypes

NS- non-significant, All values are calculated in triplicate and are expressed in terms of means \pm SD 
The ash content (Table 2) in local land races showed a wide variation, least was observed in Lakmapur local $(1.53 \%)$ and highest in Madabavi local $(9.29 \%)$. In released and advanced rabi genotypes, ash content ranged between 1.60 (DSV-5) and 8.10 per cent (SVD-0765) and in kharif grown genotypes ash content was least in SPV-2250 (1.60\%) and highest in SPV-2172 (5.61 \%). Rabi released and advanced (5.65\%) genotypes showed significantly higher content of ash compared to local land races and kharif genotypes of sorghum (Fig 1). Kadam et al., (2007) reported ash content was more in rabi (9.8 to $10.9 \%$ ) grown genotypes of sorghum compared to kharif (4.6\%) grown due to environmental influence on grains.

Grain iron and zinc contents of sorghum genotypes on dry weight basis are presented in table 3. Twenty four sorghum genotypes exhibited highly significant $(\mathrm{p}<0.001)$ variation in iron and zinc contents. Local genotypes of sorghum varied significantly for iron content $(\mathrm{p}<0.001)$ the values ranged between $4.99 \mathrm{mg} / 100 \mathrm{~g}$ (Lakmapur local) and $18.37 \mathrm{mg} / 100 \mathrm{~g}$ (Giddamaldandi). In case of rabi released and advanced genotypes significant variation $(p<0.001)$ was found, lowest was in 4.21 (Muguti) and highest was in $16.43 \mathrm{mg} / 100 \mathrm{~g}$ (Annigeri). In kharif grown genotypes iron content ranged between 4.82 $\mathrm{mg} / 100 \mathrm{~g}$ (SPV-2172) and $12.85 \mathrm{mg} / 100 \mathrm{~g}$ (SPV-2250). Over all, rabi local land races $(10.92 \mathrm{mg} / 100 \mathrm{~g})$ showed significant higher content of iron in grain compared to rabi released and advanced and kharif grown genotypes (Fig 1). Thus indicating season and genotype may influence the micronutrient content of grain. Similar variation in iron content was (0.90 to $20 \mathrm{mg} / 100 \mathrm{~g})$ also observed by Subramanian and Jambunathan (1980) due to inherent grain ash content with genotypic variability. The zinc content in sorghum genotypes rabi grown local genotypes of sorghum ranged between 0.44 $\mathrm{mg} / 100 \mathrm{~g}$ (Madabavi local) and $1.70 \mathrm{mg} / 100 \mathrm{~g}$ (Kodamurki). Whereas in released and advanced rabi genotypes zinc was between $0.49 \mathrm{mg} / 100 \mathrm{~g}$ (SVD-0813) and $1.49 \mathrm{mg} / 100 \mathrm{~g}$ (SPV-2217) and in kharif genotypes zinc was highest in SPV-2250 (1.25 mg/100g) and lower in SPV-2172 $(0.84 \mathrm{mg} / 100 \mathrm{~g})$. Among all the three types of sorghum genotypes, kharif genotypes showed highest mean value $(1.04 \mathrm{mg} / 100 \mathrm{~g})$ compared to rabi grown genotypes (Fig 1). Nguni et al., (2012) reported sorghum grain zinc ranged from 2.3 to $5.5 \mathrm{mg} / 100 \mathrm{~g}$. Our estimated zinc values are lower than the reported values it may be due to varietal difference of sorghum grain, growing conditions of the crop and soil zinc content. The wide range in composition of trace elements indicated that sorghum is a good source of minerals. Grain iron and zinc contents higher than $5 \mathrm{mg} / 100 \mathrm{~g}$ and 3.7 $\mathrm{mg} / 100 \mathrm{~g}$ respectively have been recommended for potential sorghum lines for use in the breeding programme for grain micronutrient enrichment (Kumar et al., 2013). The results indicate that there is significant variation in iron and zinc content in all the three groups.

In conclusion, the physical characteristics of grains of all sorghum genotypes when analyzed, rabi sorghum genotypes showed higher hundred kernel weight, volume, density, colour and size when compared to kharif genotypes. Similarly chemical components viz., moisture, protein, ash and mineral content of grains of sorghum genotypes differed significantly, among the rabi and kharif sorghum genotypes rabi genotypes showed higher moisture, ash and minerals like iron content except protein and zinc content. The study concludes that variations in physical characteristics and important nutrients like moisture, protein, ash and the iron and zinc contents in sorghum grains of different genotypes of different season were varied significantly. 


\section{References}

Anonymous, 2010, Official Methods of Analysis of the Association of Official Analytical Chemists, Washington, pp. 579.

Gassem, M. A. and Osman, M. A., 2003, Proximate composition and the content of sugars, amino acids and antinutritional factors of three sorghum varieties. Res. Bult., No. (125), Agric. Res. Center, King Saud Univ., 5-19.

Geeta, N., Labuschagne, M. T., Osthoff, G., Hugo, A. and Bothma, C., 2005, Physical and chemical properties associated with food quality in sorghum. African J. Plant Soil, 22: 175179.

Gopalan, C., Ramasastri, B. V. and Balasubramanian, S. C., 2010, Nutritive Value of Indian Foods. National Institute of Nutrition, Indian Council Med. Res., Hyderabad, India.

Kadam, D. E., Kulkarni, V. M., Katule, B. K. and Patil, S. V., 2007, combining ability studies in rabi sorghum (Sorghum bicolor (L.) Moench) under rainfed condition. Advances in Pl. Sci., 20(1):39-41.

Kumar, A., Reddy, B. V., Ramaiah, B., Sahrawat, K. L. and Pfeiffer, W. H., 2013, Genetic variability and character association for grain iron and zinc contents in sorghum germ plasm accession and commercial cultivars, European J. Pl. Sci. Biotechnol., 6(1): 123-154.

Mishra, B. K. and Gupta, R. K., 1995, Protocol for evaluation of wheat quality. Technoiogical. Bulletin No. 3, Directorate of Wheat Research, Karnal, India.

Nguni, D., Geleta, M., Hofvander, P., Fatih M. and Bryngelsson T, 2012, Comparative genetic diversity and nutritional quality variation among some important Southern African sorghum accessions. Australian J. Crop. Sci., 6(1): 56-64.

Samdhur, M. Y., Prabhakar, Hariprasanna, K., Mukesh, P. and Patil, J. V., 2013, combining ability studies in rabi sorghum [Sorghum bicolor (L.) Moench]. 334.

\section{How to cite this article:}

Chaitanya Itagi and Hemalatha, S. 2017. Variability in Grain Physico-Chemical Composition in Different Sorghum [Sorghum bicolor (L.) Moench] Genotypes. Int.J.Curr.Microbiol.App.Sci. 6(7): 2610-2618. doi: https://doi.org/10.20546/ijcmas.2017.607.368 